\title{
SENTIDO DE VIDA EN PACIENTES DIAGNOSTICADOS CON CÁNCER
}

\author{
Julio César Huamani Milagros Arohuanca \\ https://orcid.org/0000-0001-8159-803X \\ Universidad Ciencias de la Salud, Arequipa, Perú \\ Recibido: 10 de abril del 2019 / Aceptado: 14 de julio del 2019 \\ doi: 10.26439/persona2019.n022(2).4566
}

\begin{abstract}
Resumen: El objetivo del estudio fue analizar la percepción sobre el sentido de vida en pacientes, según tipo de cáncer, estadio de la enfermedad, tiempo de diagnóstico, grado de instrucción, sexo y edad. La muestra estuvo conformada por cien pacientes cuyo promedio etario fue de 48 años $(3,7)$, de los cuales el $72 \%$ fueron mujeres y el 28 $\%$ varones. El tipo de cáncer predominante fue mama (26\%), cuello uterino (23\%), y estómago (8\%). El cáncer en segundo grado se presentó en la mitad de los pacientes. Se utilizó el Test de Sentido de Vida o Prueba de los Propósitos Vitales (PIL), adaptación argentina realizada por Gottfried (2016). Se concluye que en los pacientes investigados predomina el logro de sentido medio y bueno, y la neurosis o depresión noógena solo se presenta en el $15 \%$ de ellos; asimismo, en el factor Actitud ante la Muerte los pacientes con cáncer de osteosarcoma de pulmón presentan rangos promedios bajos.
\end{abstract}

Palabras clave: sentido de vida / tipo de cáncer / estadio de cáncer/ edad / sexo

\section{MEANING OF LIFE AMONG PATIENTS DIAGNOSED WITH CANCER}

Abstract. This study aimed to analyze the meaning of life among cancer patients, according to the type of cancer, stage of the disease, time of diagnosis, education level, sex and age. The sample consisted of 100 patients whose average age was 48.4 years, out of which $72 \%$ were females and $28 \%$ were males. The predominant types of cancer were breast cancer $(26 \%)$, cervical cancer (23\%) and stomach cancer ( $8 \%$ ). Fifty percent $(50 \%)$ of the patients were diagnosed with stage 2 cancer. The research used the Purpose in Life (PIL) test, the Argentine adaptation of which was performed by Gottfried (2016). It is concluded that medium and good levels of meaning of life prevailed among research subjects, and noogenic neurosis or depression only occurred in $15 \%$ of such population. However, patients with osteosarcoma and lung cancer, those diagnosed with stage 3 .

Keywords: meaning of life / cancer type / cancer stage / age / sex 


\section{INTRODUCCIÓN}

Casciato y Lowitz (1990) mencionan que el 85 \% de todas las neoplasias están relacionadas con: el hábito de fumar (35\%), factores dietéticos (50\%), exposición a agentes carcinógenos $(5 \%$ ) y causas desconocidas (10 \%), que pueden deberse a virus, factores genéticos o mutaciones espontáneas.

En nuestro país, tal como menciona el diario Gestión (2016), según la Liga de Lucha contra el Cáncer esta enfermedad es la segunda causa de muerte: cada año se presentan cerca de 47000 nuevos casos de cáncer y más de 25000 peruanos fallecen a causa de la enfermedad debido a la falta de cultura preventiva. Asimismo, detalló que en el Perú los tipos de cáncer más frecuentes en mujeres son el de cuello uterino, seguido por el de mama, mientras que en los varones los más comunes son el de próstata, seguido por el de estómago.

Según Harrison (1991), el cáncer se define como aquellas malformaciones que se dan en las células; es decir, como todo ente "maligno". Se caracteriza por clonación, automatismo, anaplasia y metástasis, y genera en el paciente múltiples pérdidas y reacciones o trastornos emocionales con diversa sintomatología (Casciato y Lowitz, 1990), así como también presencia de mutismo, entendida como la negación o evasión sobre la enfermedad, acompañada de desesperanza que no solo se manifiesta durante la enfermedad, sino que ha sido una constante en la vida del afectado desde antes de enfermar (Glöecker, 2000). Así, de todas las catástrofes naturales que pueden llegar a sucedernos, la más desastrosa de todas es nuestro propio comportamiento. Solo será posible vencer el cáncer cuando aprendamos a controlarnos a nosotros mismos (Casciato y Lowitz, 1990), como ya en algún momento lo había referido Frankl (2003), aduciendo que el hombre tiene la capacidad de poder transformar la tragedia en una fuente de sentido.

El sentido de vida se ha considerado como un factor importante para las personas y para el afrontamiento de situaciones estresantes, la enfermedad, el sufrimiento y la muerte desde perspectivas teóricas de tipo existencial y cognitivo, tal como señalan Davis, Nolen-Hoeksema y Larson (1998). Frankl (1988, 1990), Janoff-Bulman (1992), Moos y Schaefer (1986) o Taylor (1983). Por otro lado, varios autores mencionan en que el hallar un sentido a la vida en una situación adversa influye en la forma de afrontar dicha situación (Steeves, 1992; Barkwell, 1991, Lipowski, 1970; Fife, 1994), ya que el sentido propio que cada persona encuentra a su enfermedad influirá en su bienestar psicológico (Lewis, 1989), por lo que este sentido llevará a la persona con cáncer a desarrollar un sentido de coherencia en relación con las experiencias que vive (O'Connor, Whicker y Germino, 1990). Esto quiere decir que el sentido de vida de una persona en fase terminal, o con algún tipo de neoplasia, solo se reestructura en la medida en que la persona pueda encontrar un sentido de vida en el sufrimiento. Las personas en esta situación atraviesan 
por situaciones donde se confrontan con experiencias límites, lo que les permite de forma directa "interiorizar" la enfermedad y todo lo que con ella llega (Jaspers, 1983).

En este sentido, los estudios realizados por Acklin, Brown y Mauger (1983) en 43 enfermos oncológicos encontraron que un "sentido trascendente de la vida", como la religiosidad y la práctica religiosa, se asociaba con un mejor afrontamiento y bienestar psicológico. De la misma manera, Lewis (1989) encontró que altas puntuaciones en el test PIL correlacionaban con baja ansiedad y buena autoestima. En la medida en que la persona atribuía sentido a su enfermedad, había menor ansiedad y mejor autoestima. Por otro lado, Meraviglia (2004), en un estudio con sesenta enfermos de cáncer de pulmón, encontró que había relación entre mayor sentido de la vida y mayor bienestar psíquico.

Con relación a todo esto, Lazarus y Folkman (1986) aducen que el afrontamiento para una enfermedad terminal requiere esfuerzos cognitivos y conductuales que son constantemente cambiantes debido a las sensaciones contradictorias que sufre el paciente y que resultan estresantes. Esto dependerá del nivel y tipo de enfermedad diagnosticado al paciente, así como de la amenaza que supone sobre sus objetivos de vida (en relación con el sentido de su vida), sus actitudes culturales y religiosas, el apoyo afectivo del que dispone, sus potencialidades para la rehabilitación psíquica y psicológica, su personalidad previa y sus estilos de afrontamiento (Holland, 1991). En tal sentido, Siegel (1995) hace hincapié en el hecho de que la persona diagnosticada de cáncer puede redefinir su actitud en tanto que esta representa un reto muy grande, pero no invencible. En la misma línea, Barthe (1997) menciona que esto debería representar un reto en la vida más que una derrota. No obstante, el paciente suele recibir el diagnóstico con una actitud mejor de la que esperan sus familiares y los médicos, pues muchas veces le permite encontrar un mayor sentido a lo que le sucede (Poveda, 1990).

Según los estudios comparativos realizados por McCorkle y Quint-Benoliel (1983) en 56 pacientes con cáncer de pulmón y 65 pacientes que habían padecido un infarto agudo, los pacientes con cáncer al pulmón mostraban más síntomas de estrés que los que habían sufrido un infarto. Quizás esta actitud se vea influida por la fase terminal de la persona con algún tipo de neoplasia debido a los síntomas ansiosos, depresivos o a la frustración de su proyecto de vida, lo que hace difícil sobrellevar la situación de la enfermedad (Matías et al., 1995). Aun así, ante las expectativas adversas que pueda tener la muerte, muchas personas cambian los criterios con los cuales estaban tomando su vida, viéndola de una manera muchos más productiva y positiva (Salmón, Manzi y Valori, 1996), a raíz de la toma de conciencia real de la muerte y por sus actitudes ante ella (Massie y Holland, 1990). En este sentido, tal como afirma Taylor (1995), buscar sentido a la vida tendría que ver con el esfuerzo por comprender la situación por la que uno atraviesa para poder integrar estas experiencias a la nueva realidad que aparece; en este caso, el padecer de cáncer. 
Para Frankl (1988, 1990), el sentido tiene que ver con el propósito a realizar, que a su vez proporciona un sustento existencial a través del objetivo planteado de manera responsable, donde esta búsqueda de sentido está presente -ya sea en mayor o menor grado- en todos los seres humanos, y donde unas difieren de otras, ya que descubrir cuál es el sentido en función de las diferentes situaciones de su vida mediante la comprensión de uno mismo permite saber cuál puede ser su actitud ante la vida o ante una situación determinada (Frankl, 1988, 1990; Längle, 2005). Dado que el sentido es creado por el individuo, tendría que ver con la necesidad de búsqueda de coherencia y propósito en la existencia (Yalom, 1984). Tal como afirma Frankl (1990, p. 84), "el sentido de la vida no se puede inventar, sino que tiene que ser descubierto". Así, diferentes autores han relacionado la búsqueda de un sentido de la vida con el sufrimiento, o incluso con situaciones de enfermedad o de muerte (Frankl, 1990, 1994, 1999; Lukas, 2002; Davis, Nolen-Hoeksema y Larson, 1998). Sin embargo, esta búsqueda de sentido también se ha relacionado con la libertad, pues sin libertad no es posible encontrar un sentido y actuar como consecuencia con responsabilidad ante aquel (Frankl, 1990, 1994, 1999).

Por último, Frankl (1990) menciona que "no es la duración de una vida humana en el tiempo lo que determina la plenitud de su sentido" (p. 119); "lo que carece de sentido de por sí, no lo adquiere por el hecho de que se eternice" (p. 121). No obstante, la muerte nos puede poner frente a nosotros mismos y hacernos reflexionar sobre si nuestra vida ha tenido sentido o no. Tal y como señala Polaino-Lorente (1995), "la muerte es una situación en que se intensifica y agiganta - aunque solo sea por un proceso acumulativo- el sinsentido o sentido de toda nuestra pasada trayectoria biográfica personal" (p. 1209), y además, "el sentido de la muerte se explica por el sentido de la vida, al mismo tiempo que contribuye al esclarecimiento de esta última" (p. 1210). Por todo lo expuesto anteriormente, el paciente neoplásico debe objetivar su realidad concreta de ese momento, ya que ayudar a los pacientes con cáncer a encontrar sentido en su experiencia parece ser un punto importante de atención para la recuperación de este (Ersek y Ferrell, 1994). Incluso hay autores que afirman que una meta del tratamiento debería ser promover la búsqueda de experiencia de sentido en los pacientes y en sus cuidadores (Ferrell et al., 2003).

El objetivo de la investigación es analizar la percepción sobre el sentido de vida en pacientes diagnosticados con cáncer según tipo de cáncer, estadio, tiempo de diagnóstico, grado de instrucción, sexo y edad. Los resultados de la investigación podrían darnos una mayor perspectiva sobre el manejo del sentido de vida del paciente frente a su enfermedad, y por ende, permitir la elaboración de abordajes terapéuticos para buscar el mejoramiento del bienestar de los diagnosticados con cáncer. 


\section{MÉTODO}

\section{Participantes}

La muestra no probabilística estuvo conformada por cien pacientes que reciben tratamiento en el Instituto Regional de Enfermedades Neoplásica del Sur (IREN-SUR). La edad $(M=48.4 ; D E=13.7)$, en el rango de 17 a 81 años. El $72 \%$ fueron mujeres y el $28 \%$ varones, con grado de instrucción primaria (37\%), secundaria (48\%) y superior (15\%). El tipo de cáncer predominante fue de mama (26\%), cuello uterino $(23 \%)$, estómago (8\%), ganglios (7\%), hígado (6\%), osteosarcoma (6\%), próstata (5\%), leucemia (5\%), liposarcoma (4\%), tiroides $4 \%$ ), pulmón (3\%) y otros (2\%). El estadio de cáncer fue del $30 \%$ para el grado 1, $50 \%$ para el grado 2 y $20 \%$ para el grado 3 . Asmismo, el $14 \%$ fue diagnosticado hace un mes, el $20 \%$ hace once meses, el $41 \%$ hace uno a dos años y el $25 \%$ hace más de dos años.

\section{Instrumentos}

Se utilizó el Test de Sentido de Vida o Prueba de los Propósitos Vitales (PIL), nombre original, Purpose in life test (Crumbaugh y Maholick, 1969), adaptación argentina por Noblejas (2000). En la investigación se utilizó la adaptación argentina del PIL Test (Test de Sentido en la Vida) de Crumbaugh y Maholick, realizada por Gottfried (2016), que fue aplicada a una muestra de 1441 sujetos entre 15 a 79 años de ambos sexos. El instrumento evalúa una escala de tres factores: el primero, percepción de sentido, conformada por once ítems, que se refiere a la captación de sentido y percepción de progreso en su vida por medio de la libertad y la responsabilidad; el segundo, vivencia de sentido, conformada por cinco ítems, referida a la vivencia emocional (entusiasmo, placer, satisfacción y novedad en contraposición a aburrimiento, pesar, rutina y apatía), que el sujeto experimenta en su vida diaria y el tercero, actitud ante la muerte, conformada por cuatro ítems, considera la posición de la persona frente a la muerte como destino inevitable. Los rangos de puntuación (Noblejas, 2000) son: logro interior de sentido muy malo (neurosis o depresión noógena), puntuación de 0-84; logro interior de sentido malo (frustración existencial), puntuación entre 85 y 93; logro interior de sentido cercano a la frustración existencial, puntuación entre 94 y 96; logro interior de sentido medio, puntuación entre 97 y 115, y logro interior de sentido bueno: puntuación entre 115 y 140. El cálculo del coeficiente alfa de Cronbach como estimador de la confiabilidad para el presente estudio fue $\alpha=0,874$ (IC95 \%, 0,835 - 0,907).

\section{Procedimiento}

El instrumento para la recopilación de la información fue aplicado a pacientes diagnosticados con cáncer que acudían al servicio oncológico IREN-Sur, quienes fueron informados del objetivo del estudio y firmaron el consentimiento informado. 


\section{Análisis de datos}

Se exploraron los datos para analizar su distribución, la asimetría, curtosis y pruebas de normalidad evidenciaron que no existe normalidad $(p<.05)$. Se realizó el análisis descriptivo y el comparativo del sentido de vida y sus dimensiones según sexo, grado de instrucción, tipo de cáncer, estadio de cáncer, tiempo de diagnóstico y edad. Para comparar dos muestras independientes, se utilizó la $U$ de Mann-Whitney con su respectivo tamaño del efecto TE, calculó de la probabilidad de superioridad $\left(P S_{\text {est }}\right)$ las normas interpretativas son: No efecto (PSest $\leq=0.0$ ); pequeño $\left(P S_{\text {est }} \geq 0,56\right)$; mediano $\left(P S_{\text {est }} \geq 0,64\right)$ y grande $\left(P S_{e s t} \geq 0,71\right)$ (Grissom, 1994). La comparación de más de dos muestras independientes se realizó con la $\mathrm{H}$ de Kruskal-Wallis, su tamaño del efecto utilizado fue el coeficiente épsilon al cuadrado $\left(E_{R}{ }^{2}\right)$, (Tomczak y Tomczak, 2014), las normas interpretativas son: pequeño $\left(E_{R}{ }^{2} \geq 0,01\right)$; mediano $\left(E_{R}^{2} \geq 0,06\right)$ y grande $\left(E_{R}^{2} \geq 0,14\right)$ (Faul, Erdfelder, Lang y Buchner, 2007), y para establecer las correlaciones, se empleó la Rho de Spearman.

\section{RESULTADOS}

En la figura 1 se observan las categorías del sentido de vida en pacientes diagnosticados con cáncer, en las cuales el 74 \% presenta sentido de vida medio y bueno; asimismo, solo el $15 \%$ muestra neurosis o depresión noógena, y un $6 \%$ frustración existencial. Es decir que los pacientes, a pesar de tener cáncer en algún grado, no presentan un vacío existencial.

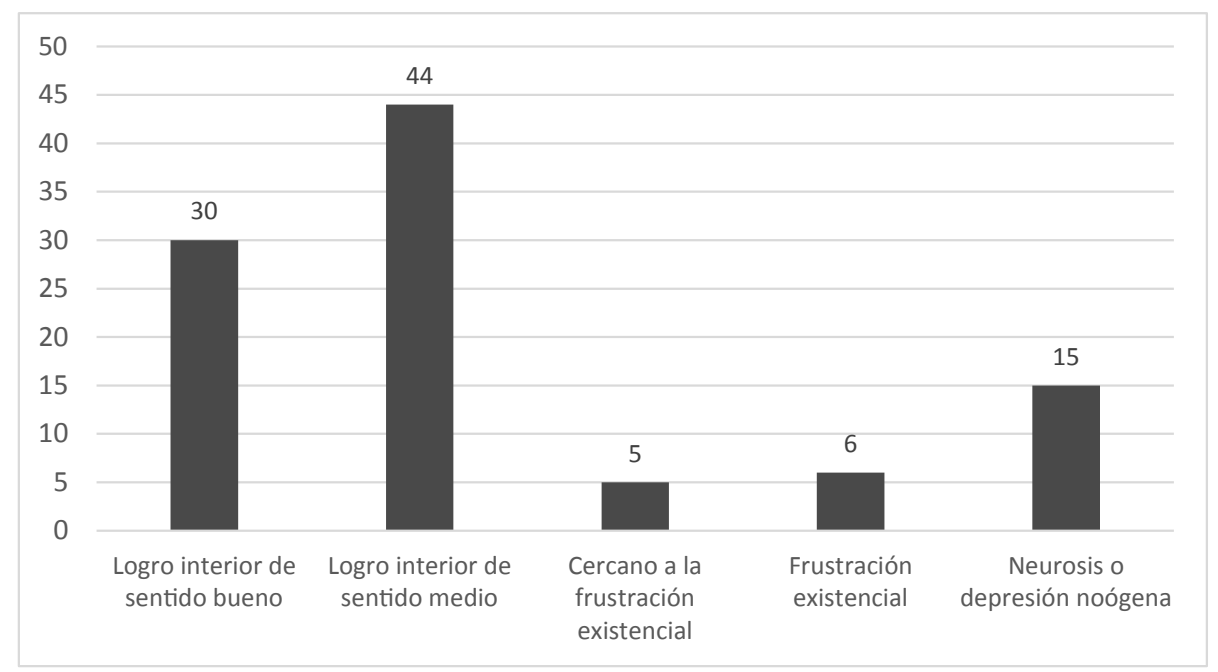

Figura 1. Sentido de vida en pacientes diagnosticados con cáncer (porcentajes) 
La tabla 1 muestra los valores descriptivos de los factores del sentido de vida en pacientes diagnosticados con cáncer. En el factor percepción de sentido, se observa que el $50 \%$ de los pacientes presenta puntajes mayores que 62 de un máximo de 76 , lo que indicaría buen sentido de vida. En el factor vivencia de sentido, el puntaje de 25 indicaría que el $50 \%$ de pacientes presenta buen sentido de vida. Asimismo, en el factor actitud ante la muerte, se observa que el $50 \%$ de los pacientes presenta puntajes mayores que 20 de un máximo de 28 , lo que lo ubicaría en un logro interior de sentido medio y cercano a la frustración existencial.

Tabla 1

Valores descriptivos de las dimensiones del sentido de vida

\begin{tabular}{llllll}
\hline & M & DE & Md & Min. & Max. \\
Percepción de sentido & 60,7 & 9,1 & 62 & 38 & 76 \\
Vivencia de sentido & 24,1 & 5,9 & 25 & 9 & 35 \\
Actitud ante la muerte & 19,7 & 4,3 & 20 & 7 & 28 \\
\hline
\end{tabular}

Al analizar el sentido de vida y sus factores según el tipo de cáncer (tabla 2), no existen diferencias estadísticamente significativas; sin embargo, al aplicar el tamaño del efecto, se observa que en el factor percepción de sentido $\left(E_{R}{ }^{2}=.04\right)$ indica un efecto pequeño (no existen diferencias en los distintos tipos de cáncer), y en el factor vivencia de sentido $\left(E_{R}{ }^{2}=.11\right)$ existe un TE mediano, lo que indicaría diferencias de la vivencia de sentido según tipo de cáncer, donde los pacientes que presentan rangos promedios bajos son los que tienen cáncer de mama, de hígado, leucemia y de pulmón. El factor actitud ante la muerte $\left(E_{R}^{2}=14\right)$ muestra TE mediano, lo que indicaría diferencias de la actitud hacia la muerte según tipo de cáncer, donde los pacientes que presentan rangos promedios bajos son los que tienen cáncer de osteosarcoma y cáncer de pulmón. Con respecto a la variable sentido de vida $\left(E_{R}{ }^{2}=.06\right)$, indican TE mediano, donde el tipo de cáncer que presenta rango promedio bajo es el de pulmón.

Al analizar el sentido de vida y sus factores según estadio de cáncer (tabla 3), no existen diferencias estadísticamente significativas; asimismo, al aplicar el tamaño del efecto, se observa que en todos los factores $\left(E_{R}{ }^{2}<0,06\right)$ indica un TE pequeño, es decir, no existen diferencias del sentido de vida y sus factores según estadio de cáncer. 


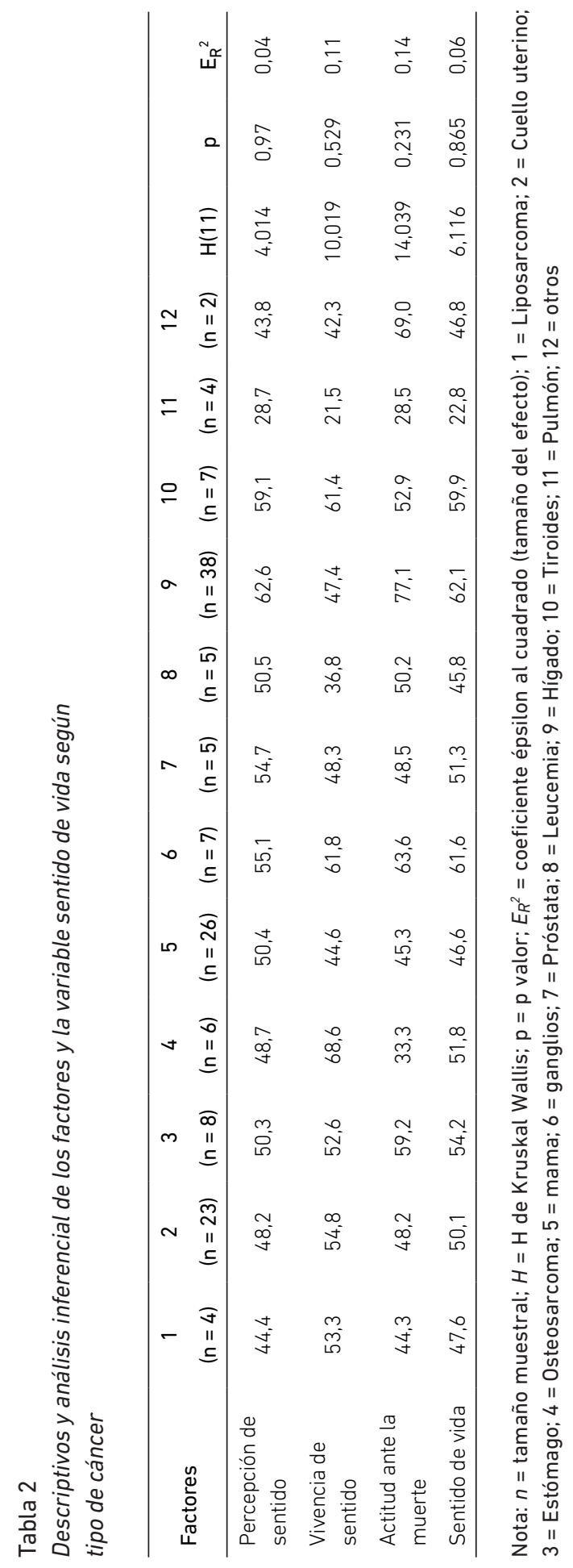


Tabla 3

Descriptivos y análisis inferencial de los factores y la variable sentido de vida según estadio de cáncer

\begin{tabular}{|c|c|c|c|c|c|c|}
\hline \multirow{3}{*}{ Factores } & Grado 1 & Grado 2 & Grado 3 & \multirow[b]{3}{*}{$H(2)$} & \multirow[b]{3}{*}{$\mathrm{p}$} & \multirow[b]{3}{*}{$\mathrm{E}_{\mathrm{R}}^{2}$} \\
\hline & $(n=30)$ & $(n=50)$ & $(n=20)$ & & & \\
\hline & Mdn(Ric') & Mdn(Ric') & Mdn(Ric') & & & \\
\hline Percepción de sentido & $65,5(10,5)$ & $62,0(13,0)$ & $62,5(15,3)$ & 0,146 & 0,930 & 0,001 \\
\hline Vivencia de sentido & $24,5(5,3)$ & $25,0(8,3)$ & $26,0(13,0)$ & 0,672 & 0,715 & 0,01 \\
\hline Actitud ante la muerte & $21,0(7,0)$ & $20,0(5,3)$ & $19,0(7,3)$ & 0,898 & 0,638 & 0,01 \\
\hline Sentido de vida & $106,5(22,0)$ & $105,5(20,5)$ & $105,5(25,5)$ & 0,039 & 0,981 & 0,001 \\
\hline
\end{tabular}

Nota: $n=$ tamaño muestral; $H=\mathrm{H}$ de Kruskal Wallis; $p=p$ valor; $E_{R}{ }^{2} .=$ coeficiente épsilon al cuadrado (tamaño del efecto).

Al analizar el sentido de vida y sus factores según tiempo de diagnóstico de cáncer (tabla 4), no existen diferencias estadísticamente significativas; asimismo, al aplicar el tamaño del efecto, en todos los factores $\left(E_{R}{ }^{2}<0,06\right)$ se indica un TE pequeño, es decir, no existen diferencias del sentido de vida y sus factores según el tiempo en que se haya dado el diagnóstico.

Tabla 4

Descriptivos y análisis inferencial de los factores y la variable sentido de vida según tiempo de diagnóstico

\begin{tabular}{|c|c|c|c|c|c|c|c|}
\hline \multirow[t]{3}{*}{ Factores } & $\begin{array}{c}\text { Menos } \\
\text { de un mes }\end{array}$ & $\begin{array}{c}\text { De } 2 \text { a } 11 \\
\text { meses }\end{array}$ & $\begin{array}{l}\text { De } 1 \text { a } 2 \\
\text { años }\end{array}$ & $\begin{array}{c}\text { Más } \\
\text { de } 2 \text { años }\end{array}$ & & & \\
\hline & $(n=14)$ & $(n=20)$ & $(n=41)$ & $(n=25)$ & & & \\
\hline & Mdn(Ric') & Mdn(Ric') & Mdn(Ric') & $\operatorname{Mdn}\left(\mathrm{Ric}^{\prime}\right)$ & $H(3)$ & $\mathrm{p}$ & $\mathrm{ER}^{2}$ \\
\hline $\begin{array}{l}\text { Percepción de } \\
\text { sentido }\end{array}$ & $64,0(11,5)$ & $60,0(14,8)$ & $63,0(15,5)$ & $61,0(9,0)$ & 2,222 & 0,528 & 0,02 \\
\hline Vivencia de sentido & $26,0(5,3)$ & $24,5(9,0)$ & $24,0(8,0)$ & $27,0(11,5)$ & 1,07 & 0,784 & 0,01 \\
\hline $\begin{array}{l}\text { Actitud ante la } \\
\text { muerte }\end{array}$ & $22,5(5,5)$ & $20,0(6,0)$ & $19,0(5,0)$ & $20,0(7,5)$ & 4,068 & 0,254 & 0,04 \\
\hline Sentido de vida & $113,0(22,0)$ & $107,5(30,3)$ & $105,0(23,0)$ & $\begin{array}{l}107,0 \\
(15,0)\end{array}$ & 1,384 & 0,709 & 0,01 \\
\hline
\end{tabular}

Nota: $n=$ tamaño muestral; $H=H$ de Kruskal Wallis; $p=p$ valor; $E R^{2}=$ coeficiente épsilon al cuadrado (tamaño del efecto)

Al analizar el sentido de vida y sus factores según grado de instrucción de los pacientes (tabla 5), se observa que no existen diferencias estadísticamente significativas; 
asimismo, al aplicar el tamaño del efecto, en todos los factores $\left(E_{R}{ }^{2}<0,06\right)$ se indica un TE pequeño; es decir, el grado de instrucción no es una variable que explique diferencias del sentido de vida y sus factores en pacientes diagnosticados con cáncer.

Tabla 5

Descriptivos y análisis inferencial de los factores y la variable sentido de vida según grado de instrucción

\begin{tabular}{|c|c|c|c|c|c|c|}
\hline \multirow{3}{*}{ Factores } & Primaria & Secundaria & Superior & \multirow[b]{3}{*}{$\mathrm{H}(2)$} & \multirow[b]{3}{*}{$\mathrm{p}$} & \multirow[b]{3}{*}{$d$} \\
\hline & $(n=37)$ & $(n=48)$ & $(n=15)$ & & & \\
\hline & Mdn(Ric') & Mdn(Ric') & Mdn(Ric') & & & \\
\hline $\begin{array}{l}\text { Percepción de } \\
\text { sentido }\end{array}$ & $62,0(11,5)$ & $61,0(12,5)$ & $64,0(12,0)$ & 1,19 & 0,552 & 0,01 \\
\hline $\begin{array}{l}\text { Vivencia de } \\
\text { sentido }\end{array}$ & $26,0(12,0)$ & $24,0(6,8)$ & $27,0(7,0)$ & 3,276 & 0,194 & 0,03 \\
\hline $\begin{array}{l}\text { Actitud ante la } \\
\text { muerte }\end{array}$ & $19,0(5,0)$ & $20,0(7,8)$ & $21,0(6,0)$ & 0,15 & 0,928 & 0,001 \\
\hline Sentido de vida & $108,0(24,0)$ & $105,0(20,8)$ & $112,0(22,0)$ & 1,332 & 0,514 & 0,01 \\
\hline
\end{tabular}

Nota: $\mathrm{n}=$ tamaño muestral; $\mathrm{H}=\mathrm{H}$ de Kruskal Wallis; $\mathrm{p}=\mathrm{p}$ valor; $E_{R}{ }^{2}=$ coeficiente épsilon al cuadrado (tamaño del efecto)

Al analizar el sentido de vida y sus factores según sexo (tabla 6), se observa que no hay diferencias estadísticamente significativas; asimismo, al aplicar el tamaño del efecto, en todos los factores $\left(E_{R}{ }^{2}<0.56\right)$ se indica que no existe TE, es decir, no existen diferencias del sentido de vida y sus factores en pacientes diagnosticados con cáncer según el sexo.

Tabla 6

Descriptivos y análisis inferencial de los factores y la variable sentido de vida según grado de instrucción

\begin{tabular}{lcccccc}
\hline \multirow{2}{*}{ Factores } & Femenino & & Masculino & & & \\
\cline { 2 - 3 } & Mdn(Ric') & & & & & \\
\cline { 2 - 3 } & $(\mathrm{n}=72)$ & Mdn(Ric') & & U & & PSest \\
\hline Percepción de sentido & $61,0(12,5)$ & $63,5(9,8)$ & & 935,5 & 0,577 & 0,46 \\
Vivencia de sentido & $25,0(8,8)$ & $26,0(8,8)$ & & 959,5 & 0,709 & 0,47 \\
Actitud ante la muerte & $19,0(6,0)$ & $21,0(5,8)$ & & 794 & 0,099 & 0,39 \\
Sentido de vida & $105,0(22,3)$ & $112,0(21,8)$ & & 889 & 0,361 & 0,44 \\
\hline
\end{tabular}

Nota: $\mathrm{n}=$ tamaño muestral; $\mathrm{U}=\mathrm{U}$ de Mann-Whitney; $\mathrm{p}=\mathrm{p}$ valor; $P S_{\text {est }}=$ Probabilidad de Superioridad (tamaño del efecto) 
En un nivel correlacional, se observa que existe relación entre la edad y el factor actitud ante la muerte. La correlación es positiva baja, lo que indicaría que a mayor edad un sentido de vida bueno o medio y a menor edad una neurosis noógena o vacío existencial. Asimismo, los factores se relacionan positivamente de forma alta.

Tabla 7

Correlaciones del sentido de vida y sus factores con la edad

\begin{tabular}{lccccc}
\hline & Edad & $\begin{array}{c}\text { Percepción } \\
\text { de sentido }\end{array}$ & $\begin{array}{c}\text { Vivencia } \\
\text { de sentido }\end{array}$ & $\begin{array}{c}\text { Actitud } \\
\text { ante la muerte }\end{array}$ & Sentido de vida \\
\hline Edad & 1 & 1 & & & \\
$\begin{array}{l}\text { Percepción } \\
\text { de sentido }\end{array}$ & 0,001 &, $660^{* *}$ & 1 & & \\
$\begin{array}{l}\text { Vivencia } \\
\text { de sentido }\end{array}$ & $-0,080$ &, $560^{* *}$ &, $362^{* *}$ & 1 & \\
$\begin{array}{l}\text { Actitud } \\
\text { ante la muerte }\end{array}$ &, $199^{*}$ &, $934^{* *}$ &, $814^{* *}$ &, $698^{* *}$ & 1 \\
Sentido de vida & 0,029 & .029 & & \\
\hline
\end{tabular}

**. La correlación es significativa en el nivel 0,01 ( 2 colas)

*. La correlación es significativa en el nivel 0,05 (2 colas)

\section{DISCUSIÓN}

Para el presente estudio se utilizó la adaptación argentina del PIL Test (Test de Sentido en la Vida) de Crumbaugh y Maholick, realizada por Gottfried (2016). Los evaluados de la presente investigación padecen de algún tipo de cáncer, lo que ha generado en ellos un cambio en su modo de existencia, es decir, sus percepciones ante la vida han dado un giro. Así, ciertos aspectos de la vida que en su momento fueron insignificantes, ahora tienen otra tonalidad debido a su acercamiento a una enfermedad como el cáncer (Bares, Blasco y Fernández, 2003).

Este cambio en la percepción de la persona con respecto a la vida tiene su confín en el sentido de vida, en encontrar un sentido dentro de toda la adversidad para poder vivir de manera coherente entre lo real y el nuevo sentido que se descubre (Bayes, 2005). Aquella persona que encuentra este sentido vive con esa vitalidad de no morirse y sin la desesperanza de no poder curarse nunca, sino más bien entiende a la vida como un proceso en el que se tiene que vivir acorde a las circunstancias por el mismo hecho de que todo tiene un sentido en la medida en uno le dé ese sentido (Bayes, Arranz, Barbero y Barreto, 1996).

En ese sentido, lo hallado en la presente investigación muestra que un $74 \%$ de los evaluados presenta un sentido de vida medio y bueno; también se observa que un $15 \%$ 
presenta neurosis noógena, y un 6 \% frustración existencial; es decir, a pesar de tener cáncer en algún grado, los pacientes no presentan un vacío existencial. Pinquart, Frölich y Silbereisen (2007) obtuvieron puntuaciones altas en el PIL; no obstante, esta población se encontraba mayoritariamente en jóvenes. Así también, Rodríguez (2006) encontró en su muestra que, a pesar de padecer cáncer, los pacientes encuentran un sentido a su vida. Estos hallazgos se atribuirían a que la mayoría de los pacientes encuentra apoyo en el entorno donde se desenvuelven, así como apoyo familiar, factor que resulta muy importante a la hora de afrontar el cáncer (Andreu et al., 2012). Tal como se nota en los resultados hallados, a pesar de que las personas presentan algún tipo de cáncer, ellas mantienen un sentido en sus vidas, lo que coincide con lo planteado por Frankl (1988, 1990a, 1990b). Lukas (2002) manifiesta que, a pesar de las situaciones adversas, la persona siempre puede encontrar sentido a su vida. Por su parte, Yalom (1985) manifiesta que las personas que encuentran un sentido de vida en esas circunstancias afrontan con mejor actitud hacia la muerte, a diferencia de aquellas personas que no tienen un sentido de vida (Davis, Nolen-Hoeksema y Larson, 1998; Frankl, 1988, 1990b, 1999a, 1999b). Por otro lado, el hecho de que los resultados muestren a la mayoría de los pacientes con un sentido de vida bueno - a pesar de padecer su enfermedad- puede deberse al apoyo familiar y a la esperanza depositada muchas veces en sus creencias religiosas, que aumenta la capacidad de la persona en poder sobrellevar su padecimiento de manera optimista (Dull y Skokan, 1995; Davis, Nolen-Hoeksema y Larson, 1998; Mclntosh, Silver y Wortman, 1993; Strawbridge et al., 1998). Sin embargo, no existen evidencias en la investigación que puedan acreditar estos resultados.

Así también, los resultados evidencian que no hay diferencias significativas con relación al tipo de cáncer, al estadio del cáncer o al tiempo de diagnóstico del cáncer; sin embargo, al aplicar el tamaño del efecto, se observa que los pacientes que presentan rangos promedios bajos son los que tienen cáncer de mama, de hígado, leucemia y de pulmón. También se hallaron diferencias en la actitud hacia la muerte según tipo de cáncer, pues los pacientes que presentan rangos promedios bajos son los que tienen cáncer de osteosarcoma y cáncer de pulmón. Estos datos pueden deberse a que, por lo general, todo cáncer representa ante la persona un hecho doloroso y frustrante. Cicirelli (1998) menciona que depende mucho del significado que da la persona a este padecimiento y la manera como valora las cosas. Marcu (2007) refiere que el sentir cercano a la muerte permite a las personas resignificar su vida; es decir, entender que, para todos, de alguna manera la vida se acaba, por lo que es vital dar importancia a la vida que nos queda (King et al., 2009).

Con relación al cáncer de mama, Anagnostopoulos y Spanea (2005) notaron que estas mujeres presentan laceraciones en su autoimagen y su sentirse atractivas, lo que influye de alguna manera en su sentido de vida. Esto mismo se corrobora con lo encontrado por Meretoja, Leidenius, Tasmuth, Sipilä y Kalso (2014), quienes indican que estas 
mujeres padecen un dolor mayor que el resto de tipos de cáncer. Así también, Bredal, Smeby, Ottesen, Warncke y Schlichting (2014) mencionan que las mujeres que padecen cáncer de mama tienen mayores inconvenientes físicos a la hora de la recuperación quimioterapéutica.

Asimismo, el sentido de vida de pacientes con cáncer no difiere según el grado de instrucción y sexo; sin embargo, algunos autores (Carver et al., 1989; Crespo y Cruzado, 1997) notaron que, por lo general, las mujeres, al ser más emocionales, tienden a desahogarse frente a sus familiares o buscar algún tipo de apoyo, mientras que los varones tienden más al aislamiento y la búsqueda de otras formas de desahogo, como las drogas o el alcohol.

Finalmente, la edad correlaciona de forma positiva y baja con el factor actitud ante la muerte, lo que indicaría que a mayor edad el sentido de vida es bueno o medio y a menor edad hay neurosis noógena o vacío existencial. Esto contrasta con lo hallado por Sarna et al. (2005), quienes encontraron que son los jóvenes quienes padecen de sentido de vida ante una enfermedad como el cáncer (Taylor, 1993; Vickberg et al., 2001). No obstante, Pinquart, Frölich y Silbereisen (2007) notaron que eran los jóvenes quienes presentaban mayor sentido de vida. Estas contradicciones encontradas, así como el estudio de esta variable en este grupo poblacional, deben ser asociadas con otras variables, como religión, desesperanza, etc. Asimismo, es necesario hacer estudios longitudinales para evidenciar si la variable sentido de vida cambia o se mantiene estable desde su diagnóstico.

\section{REFERENCIAS}

Acklin, M., Brown, E. y Mauger, P. (1983). The Role of Religious Values in Coping with Cancer. Journal of Religion and Health, 22(4), 322-332.

Anagnostopoulos, F. y Spanea, E. (2005). Assessing illness representations of breast cancer: A comparison of patients with healthy and benign controls. Journal of Psychosomatic Research, 58(4), 327-334.

Andreu, Y., Galdón, M. J., Durá, E., Martínez, P., Pérez, S. y Murgui, S. (2012). A longitudinal study of psychosocial distress in breast cancer: Prevalence and risk factors. Psychology \& Health, 27(1), 72-87.

Bares, M., Blasco, T. y Fernández, C. (2003). La inducción de sensación de control como elemento fundamental de la eficacia de las terapias psicológicas en pacientes de cáncer. Anales de Psicología, 19(2), 235-246.

Barkwell, D. (1991). Ascribed meaning: A critical factor in coping and pain attenuation in patients with cancer relating pain. Journal of Palliative Care, 7(3), 5-14.

Barthe, E. (1997). Cáncer: enfrentarse al reto. Barcelona: Robin Book. 
Bayes, R. (2005). Psicología y cuidados paliativos. Medicina paliativa, 12(3), 137-138.

Bayes, R., Arranz, P., Barbero, J. y Barreto, P. (1996). Propuesta de un modelo integral para una intervención terapéutica paliativa. Medicina paliativa, 3(3), 114-121.

Bredal, I. S., Smeby, N. A., Ottesen, S., Warncke, T. y Schlichting, E. (2014). Chronic pain in breast cancer survivors: comparison of psychosocial, surgical, and medical characteristics between survivors with and without pain. Journal of Pain and Symptom Management, 48(5), 852- 862.

Cáncer en Perú: el $85 \%$ de casos se detectan en estadios avanzados ( 5 de febrero del 2013). Gestión. Recuperado de https://gestion.pe/tendencias/cancer-peru-85casos-detectan-estadios-avanzados-144978

Carver, C., Scheier, M. y Weintraub, J. (1989). Assessing coping strategies: a theoretically based approach. Journal of Personality and Social Psychology, 56(2), 267-283.

Casciato, D. y Lowitz, B. (1990). Manual de oncología clínica. Barcelona: Salvat.

Cicirelli, V. G. (1998). Personal meanings of death in relation to fear of death. Death Studies, 22(8), 713-733.

Crespo, M. y Cruzado, J. (1997). La evaluación del afrontamiento: adaptación española del cuestionario COPE con una muestra de estudiantes universitarios. Análisis y Modificación de Conducta, 23(92), 798-830.

Crumbaugh, J. y Macholick, L. (1969). Manual of instruction for the purpose in life Test. Saratoga: Viktor Frankl Institute.

Davis, C., Nolen-Hoeksema, S. y Larson, J. (1998). Making sense of loss and benefiting from the experiencie: two construals of meaning. Journal of Personality and Social Psychology. 75(2), 561-574.

Dull, V. y Skokan, L. (1995). cognitive model of religion's influence on health. Journal of Social Issues, 51(2), 49-64.

Ersek M. y Ferrell B. (1994). Providing relief from cancer pain by assisting in the search for meaning. Journal of Palliative Care, 10(4), 15-22.

Faul, F., Erdfelder, E., Lang, A.G. y Buchner, A. (2007). G*Power 3: A flexible statistical power analysis program for the social, behavioral, and biomedical sciences. Behavior Research Methods, 39(2), 175-191.

Ferrell, B., Smith, S., Juarez, G. y Melancon, C. (2003). Meaning of illness and spirituality in ovarian cancer survivors. Oncology Nursing Forum, 30(2), 249- 258.

Fife, B. (1994). The conceptualization of meaning in illness. Social Science \& Medicine, 38(2), 309-316.

Frankl, V. (2003). El hombre doliente. Fundamentos antropológicos de la psicoterapia. Barcelona: Herder. 
Frankl, V. (1988). El hombre en busca de sentido. Barcelona: Herder.

Frankl, V. (1990a). Psicoanálisis y existencialismo: de la psicoterapia a la logoterapia. México, D. F.: Fondo de Cultura Económica.

Frankl, V. (1990b). El hombre doliente. Barcelona: Herder.

Frankl, V. (1994). La voluntad de sentido. Barcelona: Herder.

Frankl, V. (1999a). El hombre en busca de sentido último. Barcelona: Paidós.

Frankl, V. (1999b). La idea psicológica del hombre. 6a edición. Madrid: Rialp, Biblioteca del Cincuentenario.

Glöeckler, M. (2000). El cáncer, su tratamiento con la medicina antroposófica. Buenos Aires: Antroposófica.

Gottfried, A. E. (2016). Adaptación argentina del PIL Test (Test de Sentido en la Vida) de Crumbaugh y Maholick. Revista de Psicología, 12(23), 49-65. Recuperado de http://bibliotecadigital.uca.edu.ar/repositorio/revistas/adaptacion-argentinapil-test.pdf

Grissom, R.J. (1994). Probability of the superior outcome of one treatment over another. Journal of Applied Psychology, 79, 314-316.

King, L., Hicks, J. y Abdelkhalik, J. (2009). Death, life, scarcity, and value: an alternative perspective on the meaning of death. Psychological Science, 20(12), 1459-1462.

Harrison (1991). Principios de medicina interna. México, D. F.: McGraw-Hill.

Holland, J. (1991). Progress and challenges in psychosocial and behavioural research in cancer in the twentieth century. Cancer, 67(3), 767-773.

Janoff-Bulman, R. (1992). Shattered assumptions: Towards a new psychology of trauma. New York: Free Press.

Jaspers, K. (1983). Filosofía de la existencia. Barcelona: Planeta Agostini.

Längle, A. (2005). The search for meaning in life and the existential fundamental motivations. Existential Analysis, 16(1), 2-14.

Lazarus, R. y Folkman, S. (1986). Estrés y procesos cognitivos. Barcelona: Martínez Roca.

Lewis, F. (1989). Attributions of control, experienced meaning and psychosocial wellbeing in patients with advanced cancer. Journal of Psychosocial Oncology, 7(1-2), 105-119.

Lipowski, Z. (1970). Physical illness, the individual and the coping processes. Psychiatry in Medicine, 1(3), 91-102.

Lukas, E. (2002). También tu sufrimiento tiene sentido. Alivio en las crisis a través de la logoterapia. México, D. F.: LAG, Colección Sentido.

Marcu, O. (2007). Meaning making and coping: making sense of death. Cognition, Brain \& Behavior, 11(2), 397-416. 
Massie, M. y Holland, J. (1990). Depression and the cancer patient. Journal of Clinical Psychiatry, 51, 12-17.

Matías, J., Manzano, J., Montejo, A., Llorca, G. y Carrasco, J. (1995). Psicooncología: Ansiedad. Actas Luso-Españolas Neurología Psiquiatría, 23(6), 305-309.

Mccorkle, R. y Quint-Benoliel J. (1983). Symptom distress, current concerns and mood disturbance after diagnosis of life-threatening disease. Social Science \& Medicine, 17(7), 431-8.

Mcintosh, D., Silver, R. y Wortman, C. (1993). Religion's role in adjustment to a negative life event: Coping with the loss of a child. Journal of Personality and Social Psychology, $65,812-821$.

Meraviglia, M. (2004). The Effects of Spirituality in Well-Being of People with Lung Cancer. Oncology Nursing Forum, 31(1), 89-94.

Meretoja, T. J., Leidenius, M. H. K., Tasmuth, T., Sipilä, R. y Kalso, E. (2014). Pain at 12 months after surgery for breast cancer. JAMA, 311(1), 90-92.

Moos, R. y Schaefer, J. (1986). Life transitions and crisis: A conceptual overview. Coping with life crises: An integrated approach. New York: Plenum Press.

Noblejas, M. A. (2000). Fiabilidad de los tests PIL y Logotest. Nous. Boletín de Logoterapia y Análisis Existencial, 4, 81-90.

O'Connor, A., Whicker, C. y Germino, B. (1990). Understanding the cancer patient's search for meaning. Cancer Nursing, 13, 167-175.

Pinquart, M., Frölich, C. y Silbereisen, R. (2007). Change in psychological resources of younger and older patients during chemotherapy. Psycho-Oncology, 16 (7), 626-633.

Polaino-Lorente, A. (1995). La familia y la sociedad frente al enfermo terminal. El duelo en el ámbito oncológico. El sentido del sufrimiento y de la muerte. Madrid: Panamericana.

Poveda, J. (1990). Valor terapéutico de la información al paciente oncológico terminal como terapia de apoyo y su cuantificación. Tesis doctoral. Facultad de Medicina. Universidad Autónoma de Madrid. Recuperado de https://repositorio.uam.es/ bitstream/handle/10486/394/21771_poveda_de_agustin_jesus.pdf?sequence $=1 \&$ isAllowed $=y$

Salmon, P., Manzi, F. y Valori, R. (1996). Measuring the Meaning of Life for Patients with Incurable Cancer: The Life Evaluation Questionnaire (LEQ). European Journal of Cancer, 32(5), 755-760.

Sarna, L., Brown, J., Cooley, M., Williams, R., Chernecky, C., Padilla, G. y Danao, Ll. (2005). Quality of life and meaning of illness of women with lung cancer. Oncology Nursing Forum, 32(1), 9-19.

Siegel, B. (1995). Amor medicina milagrosa. Madrid: Espasa Calpe. 
Steeves, R. (1992). Patients who have undergone bone marrow transplantation: their quest for meaning. Oncology Nursing Forum, 19(6), 899-905.

Strawbridge, W., Shema, S., Cohen, R., Roberts, R. y Kaplan, G. (1998). Religiosity buffers effects of some stressors on depression but exacerbates others. The Journal of Gerontology: Social Sciences, 53B(3), S118- S116.

Taylor, S. (1983). Adjustment to threatening events. A theory of cognitive adaptation. American Psychologist, 38(11), 1161-1173.

Taylor, E. (1993). Factors associated with meaning in life among people with recurrent cancer. Oncology Nursing Forum, 20(9), 1399-1405.

Taylor, E. (1995). Whys and Wherefores: Adult Patient Perspectives of the meaning of cancer. Seminars in Oncology Nursing, 11(1), 32-40.

Tomczak, M. y Tomczak, E. (2014). The need to report effect size estimates revisited. An overview of some recommended measures of effect size. Trends Sport Sciences, 1(21), 19-25.

Vickberg, S., Duhamel, K., Smith, M., Manne, S., Winkel, G., Papadopoulos, E. y Redd, W. (2001). Global meaning and psychological adjustment among survivors of bone narrow transplant. Psycho-Oncology, 10(1), 29-39.

Yalom, I. (1984). Psicoterapia Existencial. Barcelona: Herder.

Yalom, I. (1985). Psicoterapia existencial. Bogotá: Emece. 
